\title{
Towards Serious Perception Sensor Simulation for Safety Validation of Automated Driving - A Collaborative Method to Specify Sensor Models
}

\author{
Clemens Linnhoff ${ }^{1 *}$, Philipp Rosenberger ${ }^{1 *}$, Simon Schmidt $^{2 *}$, Lukas Elster ${ }^{1 *}$, \\ Rainer Stark ${ }^{3 *}$, and Hermann Winner ${ }^{1 *}$
}

\begin{abstract}
Perception sensor modeling is essential for the safety validation of automated driving systems in virtual environments. Nevertheless, the community lacks a methodical approach to derive requirements for such sensor models that enables a serious application for safety validation in the first place. This article provides a method to specify sensor models for the environmental perception of automated driving systems. The key of the approach is a collaborative collection of causeeffect chains as the basis for specification. With this collection at hand, a tabular form is introduced to extract the relevance of the effect chains to be modeled. Combined profound expert assessments in the table enable the test engineer to specify sensor models within a traceable decision-making process.
\end{abstract}

\section{INTRODUCTION}

The development of automated driving systems is becoming increasingly virtualized with the help of simulated vehicle environments. The growing complexity of automated driving systems, in the way of increased connectivity and distributed information processing, is the underlying reason. In addition, there is an enormous testing effort, which cannot be fulfilled in the classical way, by means of prototypes, in terms of time and cost [1]. During the system development process (SDP), sensor models are used in simulation setups for processing perceptual information from a virtual vehicle environment. They output perceptual measurement data to a fusion unit or directly to the automated driving system.

Sensor models, or more precisely their specifications, are the central subject of this publication. In the automotive industry, sensor models and driving systems are developed in parallel but often separated from each other. In most cases, several stakeholders from different domains are involved. In the course of development, sensor models and driving systems change almost continuously. In different phases of the SDP, various requirements can be derived directly from the automated driving system, the technical simulation setup or from development and test objectives. It is therefore not possible to provide a "complete" sensor model at the

This work received funding from SET Level and VVM of the PEGASUS project family, promoted by the German Federal Ministry for Economic Affairs and Energy based on a decision of the Deutsche Bundestag.

${ }^{1}$ The authors are with the Institute of Automotive Engineering, Technical University of Darmstadt, 64287 Darmstadt, Germany $<$ prename $>$. <surname $>$ atu-darmstadt. de

${ }^{2}$ Simon Schmidt is with the Volkswagen AG, Wolfsburg, Germany simon.schmidt5evolkswagen. de

${ }^{3}$ Rainer Stark is with the Department of Industrial Information Technology, Technische Universität Berlin, Berlin, Germany rainer.starketu-berlin. de

* The authors contributed equally to the work by collaboration. beginning of development, since the requirements of later phases are often only incompletely known at this point.

In practice, there are often only a few global or temporal invariant requirements, which leads to difficulties in specifying sensor models. Each of the typically involved experts represent the perspective of their individual domain, which is subjected to restrictions. To specify a sensor model, relations between the intended usage, detailed sensor knowledge and its impact on the system under test (SUT) have to be known. E.g. a modeler of a specific sensor technology, for example a lidar sensor expert, has a profound sensor insight, but is rarely able to determine the content to be modeled for the intended stimulation of the SUT. And a developer of an automated driving system has a certain expectation of the environment perception, but usually does not have the necessary knowledge of the underlying sensor principles.

\section{State OF ThE ART}

In literature, lots of different models and model types are available. Schlager et al. [2] provide an overview of the state of the art of perception sensor models. In a first approach they try to group and compare various models, but the diversity in existing models poses a challenge. Hence, there is no generally accepted classification procedure so far. Based on systematic development approaches like ModelBased Systems Engineering, models should be created and used, starting from requirements. In the discipline of sensor modeling, however, this proves difficult because there are no generally accepted requirements available [3]. Furthermore, there are hardly any generally accepted standards for perception models up to now, as described in the example of radar sensor modeling by Holder et al. [4]. Even if requirements are available in the SDP, they usually come from the modelers and not from the model users. In addition, the variety of possible modeling methods makes selection difficult, since they are often insufficiently compared. There are also no standardized benchmarks that can be used to easily compare different models. Holder [5] shows some exemplary scenarios used in practice as an initial reference.

From the practical point of view, Schäferle [6] introduces different model categories and advices of application, depending on simulation goals. Rosenberger et al. [7], on the other hand, derive the requirements for models directly from real sensors, with the goal of modeling automotive hardware and signal processing. To use the models within an SDP in the automotive industry, continuous integration is needed. 
Requirements from the SDP are changing depending on the current stage. Hence, models have to be adapted over time. A sensor model specified at the beginning of the development will not meet the requirements of later phases and must be further developed. An initial approach of integration into SDP is proposed by Schlager et al. [2] and developed further by Schmidt and Stark [8]. In order to support flexible and continuous integration, modular sensor modeling approaches are introduced by [8]-[11]. They advocate a generic and modular architecture to gain high interchangeability and create sensor models based on modular principles.

Functional decomposition is used to break down an overall sensor behavior into individually modelable effects and separate model development from parameterization effort [11]. A functional decomposition for perception sensors can be performed as shown by Holder et al. on radar [12], by Rosenberger et al. on lidar [13] and by Mohr et al. on camera [14]. They introduce functional blocks and interfaces to model a sensor type specific signal processing chain. Following a modular approach, Muckenhuber et al. [15] demonstrate how to model sensor effects. Rosenberger et al. [7] perform a systematic analysis of real lidar sensors to figure out which effects are relevant and if they could be modeled. Holder et al. [3] investigate important effects of object detection and classification on a lidar sensor using data mining methods. They also compare their modeled results with a real sensor.

Although some approaches already exist to represent specific properties of sensors in the form of modular sensor effects, it still poses a challenge since there are too many effects to completely describe them [4], [7] and the fundamental problem of application remains. It is still not clear how sensor effects have to be systematically selected and combined based on the requirements from the automotive SDP. This article will provide a systematic and collaborative approach to specify sensor models.

\section{Definition of Terms}

Usually, all introduced involved parties come from different stakeholders with different technological backgrounds, which complicates a direct and concrete discussion about modeling approaches as well as inputs and outputs of models. Therefore, a commonly agreed language is needed.

The authors introduced a naming scheme for sensor models by defining the input and output interfaces in [11] as input-based technology output model using the input and output definitions from the Open Simulation Interface (OSI) [16] and the ISO 23150 [17] international standard.

The ISO 23150 defines three possible output formats. A detection is defined as a "sensor technology specific entity represented in the sensor coordinate system based on a single measurement of a sensor". A feature is a "sensor technology specific entity represented in the vehicle coordinate system based on multiple measurements". An object is the "representation of a real-world entity with defined boundaries and characteristics in the vehicle coordinate system" [17, pp. 2-3]. As the term detection is occupied in this context, we define the process of extracting detections, features or objects from present signal or data at the sensor as identification. The identification step can also contain classification and tracking while assigning an ID. These definitions will now be used to further define the contents of the effect chains between input and output of an automotive perception sensor system model. The terms are defined with camera, lidar and radar in mind but are also applicable to other sensor technologies such as ultrasonic or GNSS.

The effect chains start with the emission (active sensors) or propagation (passive sensors) of a signal in the surroundings of the sensor. After propagation, the signal is received by the sensor and further processed in multiple signal processing steps. During emission, propagation, reception, and signal processing, the information in the signal deviates from the original information about the environment, existing as ground truth (GT) under clean room conditions. Clean room conditions describe idealized measurement conditions, without the influence of weather, dust, dirt etc. The GT is taken in this clean room environment by an omniscient observer. All effects including classifications like false positive (FP) and false negative (FN) are defined according to Nuss et al. [18] with respect to the overall GT.

Every condition leading to a deviation in the information is defined as the cause for an effect. A cause can be a property of the emission unit's hardware, like the physics of the antenna design of a radar, they can be located in the propagation path, like weather conditions, or in the signal processing, like the resolution of AD-conversion.

For an effect to appear, a signal is needed, which is defined as a quantity of energy influencing the sensor according to its measurement principle. "Signals occur naturally and they are also synthesized" [19, p.1]. In case of active sensors, signals mainly consist of energy irradiated by the sensor.

As effect, we define the deviation from the originally existing information about the environment in the signal or data. For example, an error in the measured distance to an object resulting from limited range resolution would be an effect in the signal processing of the sensor. An effect can have multiple causes but also a cause can lead to multiple effects. Consequently, a cause-effect chain can induce a subsequent effect in the signal or data.

If an effect is observable at the output of a sensor (model), we call it a phenomenon. Phenomena influence subsequent system(s) and therefore constitute the application point for defining the requirements for sensor system models. This is partly described by Riedmaier et al. [20] for object lists as an output format, where phenomena can be observed and tested with suitable metrics.

In literature, sometimes the term artifact is found. Holder [5] defines an artifact as a "noticeable deviation from GT in the sensor readings". In this case, artifacts are effects that occur in the processing chain after AD-conversion.

Usually, there is not one single cause for an effect or phenomenon, rather a cause-effect chain that leads to a deviation from GT. The cause-effect chains and their interdependencies constitute the overall modeled phenomena. 


\section{EFFECTS}

To evaluate the cause-effect chains for requirement specification, first, effects are collected and sorted. The effects can be sorted into the data processing steps, where they occur in the sensor signal [21]. While in [21] only object models are considered, we propose to take the whole processing chain from emission to object identification into account, see Tab. I. Consequently, a holistic view on modeling sensor systems, containing not only the front-end but also a complete data processing chain possibly up to object identification, is needed. The presented approach can be used to specify sensor system models, as well as models that end after the detection identification or even beforehand. This allocation facilitates modular sensor modeling for all sensor principles and modeling approaches. As the effects are defined within the real sensor's signal processing chain, the modeling approach is irrelevant for the effect definition. However, for the requirement specification, it matters at what point an effect becomes a phenomenon.

The generic decomposition of a sensor system processing chain with object output results in the blocks emission, signal propagation, reception, signal pre-processing, detection identification, feature identification and object identification. The emission step contains the generation and emission of the signal of active perception sensors as well as 3rd party signals. During signal propagation, the signal travels through the environment and possibly interacts with objects and particles. The reception comprises the reception hardware of all sensors. During pre-processing, the signal is digitized through analog-digital-conversion. This block can also include processing steps like Fast Fourier Transformations and data reduction. In the detection identification block, detections are identified by thresholding, peak detection and interpolation. Detections are further processed by filtering, pre-tracking or classification methods in the feature identification block. The object identification block contains object detection, classification and prediction steps.

Tab. I lists typical sensor effects for camera, lidar and radar, sorted into signal processing blocks. However, most of the effects do not occur independently but influence other effects in different decomposition blocks. A specular reflection during signal propagation can lead to a power value at a wrong angle during reception. During pre-processing, this error-prone angle might be amplified by limited resolution or reverted if it lies in the same resolution cell as the original angle. If not reverted, the wrong angle will also appear as a detection state error or as FP or FN during detection identification. These effects can propagate through feature identification and further result in an object existence or state error in the object identification block.

While the list of effects is quite broad, it remains a loose accumulation of effects without any claim of completeness. Therefore, the list is not sufficient for sensor modeling, but depending on the desired model fidelity, the entire causeeffect chains need to be analyzed.
TABLE I: Categorization of exemplary sensor effects for $\star$ : camera, $\nabla$ : lidar, and a: radar

\begin{tabular}{|c|c|}
\hline Block & Effect \\
\hline Emission & $\begin{array}{l}\text { Emission unit: } \\
\quad \text { optic } \nabla \text { [22, pp. 621], } \\
\text { antenna \& [23] } \\
\text { Emission power level } \nabla[24], \text { \& }[25] \\
\text { Hardware non-linearities } \nabla[26], \text { \& }[27] \\
\text { Phase noise \& [28], [29] } \\
\text { 3rd party signals: } \\
\quad \text { Other sensors } \nabla \text { [30], \& [31] } \\
\quad \text { Street, sun lights etc. } \star[32], \nabla[33]\end{array}$ \\
\hline $\begin{array}{l}\text { Signal } \\
\text { propagation }\end{array}$ & 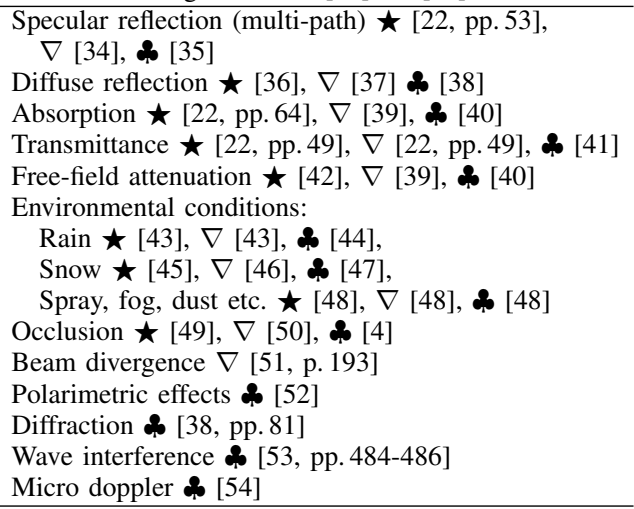 \\
\hline Reception & 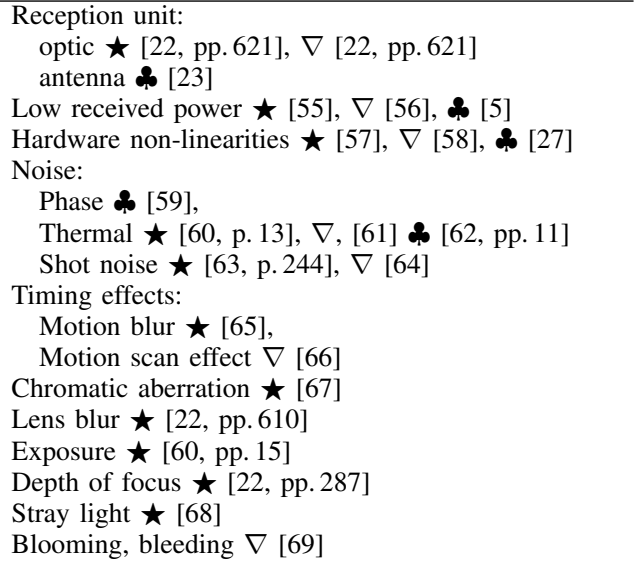 \\
\hline Pre-processing & 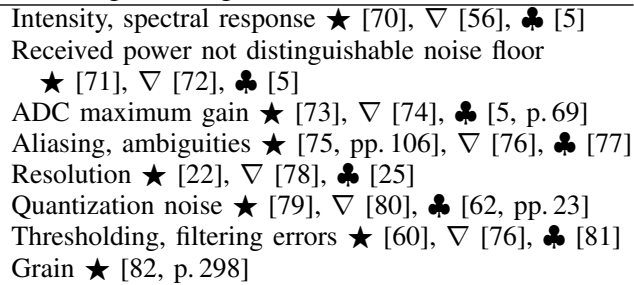 \\
\hline $\begin{array}{l}\text { Detection } \\
\text { identification }\end{array}$ & 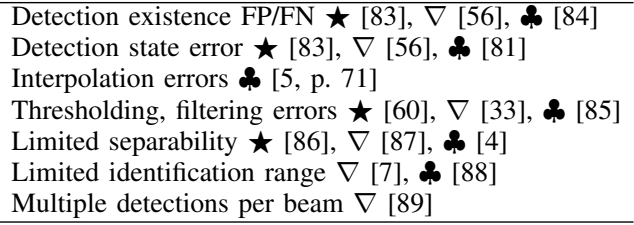 \\
\hline $\begin{array}{l}\text { Feature } \\
\text { identification }\end{array}$ & $\begin{array}{l}\text { Feature existence FP/FN } \nabla \text { [87], \& [90] } \\
\text { Feature state error } \nabla[87], \&[91] \\
\text { Thresholding, filtering errors } \nabla[87] \text {, \& [91] } \\
\text { Ambiguity resolution error \& [92] }\end{array}$ \\
\hline $\begin{array}{l}\text { Object } \\
\text { identification }\end{array}$ & $\begin{array}{l}\text { Object existence FN/FP } \star[93], \nabla[87], \boldsymbol{\&}[94] \\
\text { Object separation error } \star[95], \nabla[87], \boldsymbol{\&}[4] \\
\text { Object state error } \star[95], \nabla[87], \boldsymbol{\&}[96] \\
\text { Object classification error } \star[95], \nabla[87], \boldsymbol{\&}[94] \\
\text { Latency } \star[97], \nabla[98], \boldsymbol{\&}[97]\end{array}$ \\
\hline
\end{tabular}




\section{Methodology for Sensor Model Specification}

With the derived overview regarding effects on different sensor technologies in Tab. I, a two-stage method is developed for model specification, as described in the following.

The first step is to collect cause-effect chains until the state of the art is covered and collectively agreed on. Since effects can have multiple causes, and can be the cause for other effects, the effect chains are arranged in a tree representation. Starting with an observable effect on a high level in the signal processing chain, the level of detail of the effect's origins is systematically derived as a top-down method. An exemplary tree is shown in Fig. 1. Since a single person will not be able to generate the entire tree, it has to be collected collaboratively by the community. Therefore, we call it Perception Sensor Collaborative EfFect and Cause Tree (Percollect).

Effects are denoted as gray boxes which are connected by lines. Each connection has to be proven by referencing scientific literature, accepted by the community of the sensor domain. Instead of literature, connections can also be proven by referencing actual sensor measurements revealing the cause-effect relationship. Causes, which are not effects and which are not broken down any further, are denoted as green or blue boxes, marking the end of the respective branch.
Green boxes represent causes, which are independent of the specific sensor. Causes that are design parameters during development of the sensor are marked as blue boxes. The exemplary top of the tree is a false negative in the object list. This effect occurs, among other reasons, due to a false negative feature resulting from a false negative detection. The effect chain is followed down the signal processing steps to causes such as the emitter wavelength within the emission block. PerCollECT deviates from classic tree representations, because branches from singular causes intersect for multiple effects. The emitter wavelength, for example, is a cause for multiple effects. Its node therefore has multiple parent nodes and appears in multiple branches of the tree.

The cause-effect chains shall be as generic as possible and use case independent to keep all trees similar and comparable. Nevertheless, as the trees grow, it is expected that the effect chains will become more and more sensor specific, resulting in partly diverging tree structures per sensor technology (camera, lidar, radar, ultrasonic etc.). Potentially proposed additions and changes to the trees over time need to be maintained by the community.

Consequently, PerCollECT will be usable for every specific sensor technology. Working examples of PerCollECT can be found under https://github.com/PerColleCT.

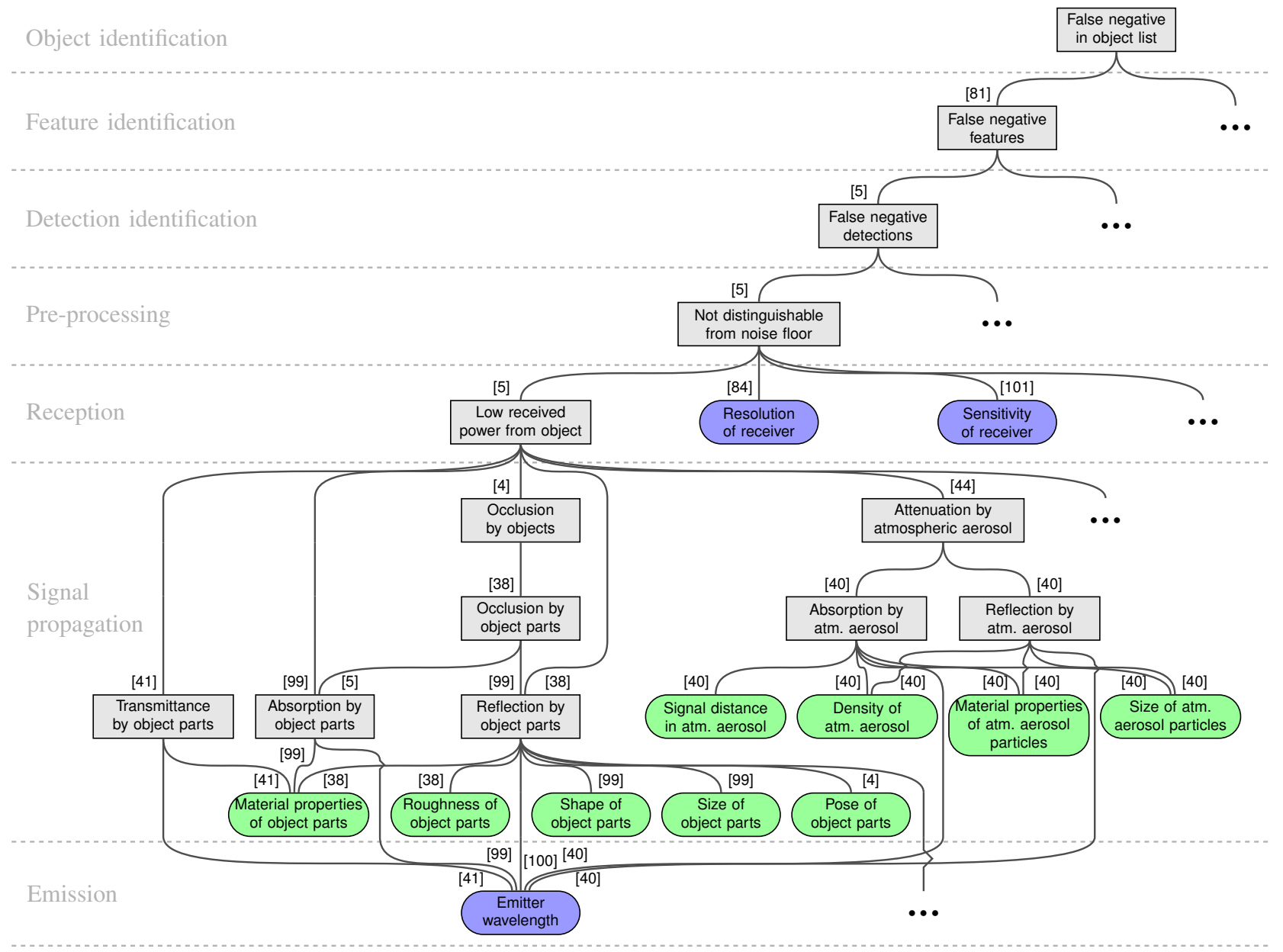

Fig. 1: Exemplary Perception Sensor Collaborative Effect and Cause Tree (PerCollECT) for active perception sensor models.

Gray rectangles: Effects; Green rounded rectangles: System independent causes; Blue rounded rectangles: Design parameter causes 
As the second step to systematically derive the model specification, a method to evaluate the relevance of individual phenomena and their effect chains within the pre-defined Operational Design Domain [102] (ODD) is introduced. We call it CAUSe, EFFECT, AND PhenOMENON RELEVANCE ANALYSIS (CEPRA). CEPRA is a descision-making tool for the test engineer, who will design the overall test setup and oversee the component integration and test execution. To derive requirements for any model or system, its intended usage must be known. The ODD determines the content of test scenarios and in consequence the environment and objects that define the conditions in which the perception sensor model is applied. Additionally, the SUT applied within that ODD determines the outputs of the model. The model outputs define the decomposition block at which effects become phenomena, while defining the starting points to be considered in PerCollECT. From there, the individual effect chains per phenomenon are extracted from PerCollECT into a table for relevance analysis, as exemplary shown in the first three columns of Tab. II.

In order to assess the relevance of these automatically extracted phenomena and effect chains within the ODD, the test engineer needs to consult two experts. First, an expert on the considered sensor technology assesses the occurrence of the individual effect chains within the ODD. While some effect chains are the dominant cause for a phenomenon within the ODD, others make only a minor contribution. This highly depends on the specific properties of the considered sensor. The sensor knowledge is documented in column four of CEPRA as a score for the occurrence. It is denoted as a value between 1 ("Cannot occur") - 10 ("Extremely high") and is based on the rank definitions for occurrence in [103, pp. 123]. The score should be accompanied by a rationale for future traceability of the decision process during the model specification. Second, an expert on the SUT is consulted to determine, which phenomena have the highest impact on the SUT within the ODD, scored between 1 ("Very low") - 10 ("Very high") according to [103, p. 122]. Every SUT depends differently on certain aspects of its input data. A detection fusion algorithm will be influenced by other effect chains than a high-level behavior planner for a highway pilot. The latter will be the example listed in Tab. II.

We propose to add these two independent expert scores, resulting in an overall relevance score for every phenomenon and effect chain. Using a sum instead of multiplication facilitates the impact of extremely low or high individual scores with respect to safety consideration. This result is the basis of decision-making for the overall model specification. Taking the result into account with the available resources and the remaining tolerable safety risk, the test engineer will define phenomena with their respective effect chains as requirements in the specification for the intended usage. Requirements need to have quantifiable values or test scenarios with pass-fail-criteria. From the identified relevant effect chains, falsification scenarios can be derived for model verification. This step, however, is not part of this work.

CEPRA can be performed in various phases of the SUT development with increasing level of detail. In early development stages, the modeling detail can be defined by setting the maximum depth of PerCollECT. The length of the effect chains is therefore limited during the extraction from PerCollECT to CEPRA, reducing the specification effort.

Performing CEPRA on the basis of PerCollECT will combine expert knowledge on sensor and SUT domains while considering the ODD to identify relevant effect chains and phenomena for the intended model usage. It will further document the decision-making process in detail, to ensure revision proof throughout the development process.

TABLE II: Exemplary Cause, Effect, and Phenomenon Relevance Analysis (CEPRA) for Sensor Modeling

\begin{tabular}{|c|c|c|c|c|c|c|c|}
\hline \multirow{2}{*}{$\begin{array}{l}\text { Pheno- } \\
\text { menon } \\
(\mathrm{P})\end{array}$} & \multirow{2}{*}{$\begin{array}{l}\text { Effect chain }(\mathrm{EC}) \\
\text { of phenomenon }\end{array}$} & \multirow{2}{*}{$\begin{array}{l}\text { Causes of } \\
\text { effect chains }\end{array}$} & \multicolumn{2}{|c|}{$\begin{array}{l}\text { P\&EC occurrence in ODD* } \\
(O, \text { filled by sensor expert })\end{array}$} & \multicolumn{2}{|c|}{$\begin{array}{l}\text { P\&EC impact on SUT in ODD* } \\
(I, \text { filled by SUT expert })\end{array}$} & \multirow{2}{*}{$\begin{array}{c}\begin{array}{c}\text { Relevance } \\
\text { of P\&EC }\end{array} \\
O+I\end{array}$} \\
\hline & & & {$[1,10]$} & Rationale & {$[1,10]$} & Rationale & \\
\hline \multirow{4}{*}{$\begin{array}{l}\text { False } \\
\text { negative } \\
\text { in } \\
\text { object } \\
\text { list }\end{array}$} & $\begin{array}{l}\rightarrow \text { FN features } \rightarrow \text { FN detections } \\
\rightarrow \text { Not dist. from noise floor } \\
\rightarrow \text { Low rec. power from object } \\
\rightarrow \text { Occlusion by objects } \\
\rightarrow \text { Occlusion by object parts } \\
\rightarrow \text { Reflection by object parts }\end{array}$ & \multirow{2}{*}{$\begin{array}{l}\text { - Materials of } \\
\text { reflect. obj. parts } \\
\text { - Roughness of } \\
\text { reflect. obj. parts } \\
\text { - Shapes of } \\
\text { reflect. obj. parts } \\
\text { - Size of } \\
\text { reflect. obj. parts } \\
\text { - Emitter wavel.** }\end{array}$} & 4 & $\begin{array}{l}\text { FN objects caused by } \\
\text { occluding reflecting objects } \\
\text { occurs rarely in a front } \\
\text { radar on a highway, because } \\
\text { of multi-path propagation. }\end{array}$ & 6 & $\begin{array}{l}\text { FN obj. occurring because } \\
\text { of occlusion in a front radar } \\
\text { have a moderate impact } \\
\text { because mainly only direct } \\
\text { neighbor objs. considered. }\end{array}$ & 10 \\
\hline & $\begin{array}{l}\rightarrow \text { FN features } \rightarrow \text { FN detections } \\
\rightarrow \text { Not dist. from noise floor } \\
\rightarrow \text { Low rec. power from object } \\
\rightarrow \text { Reflection by object parts }\end{array}$ & & 2 & $\begin{array}{l}\text { FN objects caused by compl. } \\
\text { away-reflecting obj. cannot } \\
\text { be ruled out, but are not } \\
\text { expected on highway. }\end{array}$ & 9 & $\begin{array}{l}\text { FN objects occurring } \\
\text { in a front radar } \\
\text { have a very high impact } \\
\text { on a highway pilot. }\end{array}$ & 11 \\
\hline & $\begin{array}{l}\rightarrow \text { FN features } \rightarrow \text { FN detections } \\
\rightarrow \text { Not dist. from noise floor } \\
\rightarrow \text { Low rec. power from object } \\
\rightarrow \text { Attenuation by atm. aerosol } \\
\rightarrow \text { Absorption by atm. aerosol }\end{array}$ & $\begin{array}{l}\text { - Signal dist. } \\
\text { in atm. aerosol } \\
\text { - ... } \\
\text { - Emitter wavel. }{ }^{* *}\end{array}$ & 3 & $\begin{array}{l}\text { FN objects caused by } \\
\text { completely absorbing } \\
\text { atmospheric aerosol occur } \\
\text { only in harsh weather in } \\
\text { a front radar on a highway. }\end{array}$ & 5 & $\begin{array}{l}\text { FN objects occuring } \\
\text { in harsh weather conditions } \\
\text { may be covered by safety } \\
\text { concept with a moderate } \\
\text { impact on the highway pilot. }\end{array}$ & 8 \\
\hline & $\bullet \bullet \bullet$ & & & & & & \\
\hline$\bullet \bullet \bullet$ & & & & & & & \\
\hline
\end{tabular}

Legend: Normal font: Automatically generated content from PerCollECT after sensor output definition; Italic: Expert knowledge needed * Operational Design Domain (ODD) must be defined beforehand (here: a German highway with all its elements for a highway pilot as SUT). ${ }^{* *}$ These causes are design parameters by the SUT (here: a highway pilot's radar at the front center) and must be defined beforehand. 


\section{CONCLUSION AND OUTLOOK}

In this work, a method to systematically specify perception sensor models for the development and validation of automated driving systems is introduced. The basis of the method consists of collaboratively collecting effects in the signal processing of perception sensors and linking the cause-effect chains in a sensor technology specific tree structure, we call PerCollECT. The branches of that tree are automatically extracted to a table, where every causeeffect chain is evaluated by an expert in the respective sensor technology and an expert on the SUT. These profound expert evaluations result in an overall score for every effect chain, enabling the decision-making for the test engineer. Individual phenomena with their respective effect chains will be selected as requirements for the development of the sensor models. The effect chain scoring will aid test engineers to systematically specify sensor models and at the same time ensure revision proof throughout the development process, by documenting every decision-making step during the requirement engineering phase for traceability.

The proposed method is concentrated on requirements of the phenomena in sensor data. However, some requirements are not covered by the introduced method, such as minimum execution speed, model packaging, test environment etc. These requirements need to be additionally considered by the test engineer. The approach was developed with radar, lidar and camera sensors in mind, but can be adapted for other technologies, such as ultrasonic, mobile communications and GNSS, for which similar modular simulation approaches exist [104], [105]. The strict separation of sensor technologies in camera, lidar and radar can also be adapted in the future as measurement principles combine together, e.g. 3D-ToF camera. PerCollECT can be further extended by adding real world sensor measurement data as reference for the links between effects in addition to literature sources. Adding logical gates to the links will enhance the level of detail by denoting, if all multiple causes have to exist (AND gate), or if one of the causes will suffice (OR gate) in order for the effect to appear.

Adding more columns to the introduced first version of CEPRA for the assessment of modeling complexity by the sensor expert would further aid the decision-making process. Additional columns for the evaluation from other experts, e.g. in scenario design, sensor fusion or modular safety assessment, will also enable analysis such as edge-case identification, scenario coverage, redundancy analysis of sensor setups etc. and therefore broaden the range of application even further. All these topics are currently researched by multiple publicly funded research projects. Therefore, we expect this method of collaboratively analyzing and collecting cause-effect chains with its broad range of application to become a game changer for the entire industry.

To get the collection process started, we are making PerCollECT publicly available as an open-source, collaborative, and scientific database. Be part of PerCollECT under: https://github.com/PerCollECT.

\section{REFERENCES}

[1] N. Kalra and S. M. Paddock, Driving to Safety: How Many Miles of Driving Would It Take to Demonstrate Autonomous Vehicle Reliability? Santa Monica, CA: RAND Corporation, 2016.

[2] B. Schlager, H. Holzer, M. Kirchengast, F. M. Maier, S. Muckenhuber, R. Rott, J. Ruebsam, K. Saad, S. Schmidt, G. Stettinger, and D. Watzenig, "State-of-the-art sensor models for virtual testing of ADAS/AD functions," SAE International Journal of Connected and Automated Vehicles, vol. 3, pp. 233-261, 2020.

[3] M. F. Holder, P. Rosenberger, F. Bert, and H. Winner, "Datadriven derivation of requirements for a lidar sensor model," in Graz Symposium Virtual Vehicle, 2018, p. 10.

[4] M. F. Holder, P. Rosenberger, H. Winner, T. D'hondt, V. P. Makkapati, F. M. Maier, H. Schreiber, Z. Magosi, Z. Slavik, O. Bringmann, and W. Rosenstiel, "Measurements revealing challenges in radar sensor modeling for virtual validation of autonomous driving," in 2018 21 st International Conference on Intelligent Transportation Systems (ITSC). IEEE, 2018, pp. 2616-2622.

[5] M. F. Holder, "Synthetic generation of radar sensor data for virtual validation of autonomous driving," PhD Thesis, TU Darmstadt, 2021.

[6] S. Schäferle, "How do I choose the correct sensor model for my specific application?" 2019, Accessed: 2020/10/23. [Online]. Available: https://ipg-automotive.com/uploads/tx_pbfaqtickets/files/ 98/SensorModelLevels.pdf

[7] P. Rosenberger, M. F. Holder, M. Zirulnik, and H. Winner, "Analysis of real world sensor behavior for rising fidelity of physically based lidar sensor models," in 2018 IEEE Intelligent Vehicles Symposium (IV). IEEE, 2018, pp. 611-616.

[8] S. Schmidt and R. Stark, "Der Einsatz von Sensormodellen bei der Entwicklung automatisierter Fahrfunktionen," in Conference Proceedings, NAFEMS20 Virtuelle DACH Konferenz, Fachkonferenz für Berechnung \& Simulation im Engineering, 2020.

[9] R. J. Telgarsky, M. C. Cates, C. Thompson, and J. N. Sanders-Reed, "High-fidelity ladar simulation," in Defense and Security, G. W. Kamerman, Ed., 2004, pp. 194-207.

[10] T. Hanke, N. Hirsenkorn, B. Dehlink, A. Rauch, R. Rasshofer, and E. Biebl, "Generic architecture for simulation of ADAS sensors," in Proceedings International Radar Symposium, 2015, pp. 125-130.

[11] C. Linnhoff, P. Rosenberger, M. F. Holder, N. Cianciaruso, and H. Winner, "Highly parameterizable and generic perception sensor model architecture," in 6. Internationale ATZ-Fachtagung Automated Driving, 2020

[12] M. F. Holder, Z. Slavik, and T. D'hondt, "Radar signal processing chain for sensor model development," in Validation and Verification of Automated Systems, A. Leitner, D. Watzenig, and J. IbanezGuzman, Eds. Springer International Publishing, 2020, pp. 119133.

[13] P. Rosenberger, M. F. Holder, M. R. Zofka, T. Fleck, T. D’hondt, B. Wassermann, and J. Prstek, "Functional decomposition of lidar sensor systems for model development," in Validation and Verification of Automated Systems, A. Leitner, D. Watzenig, and J. IbanezGuzman, Eds. Springer International Publishing, 2020, pp. 135-149.

[14] M. Mohr, G. Garcia Padilla, K.-U. Däne, and T. D'hondt, "Camera sensor system decomposition for implementation and comparison of physical sensor models," in Validation and Verification of Automated Systems, A. Leitner, D. Watzenig, and J. Ibanez-Guzman, Eds. Springer International Publishing, 2020, pp. 151-163.

[15] S. Muckenhuber, H. Holzer, J. Rübsam, and G. Stettinger, "Objectbased sensor model for virtual testing of adas/ad functions," in 2019 IEEE International Conference on Connected Vehicles and Expo (ICCVE), 2019, pp. 1-6.

[16] T. Hanke, N. Hirsenkorn, C. van Driesten, P. Garcia Ramos, M. Schiementz, S. Schneider, and E. Biebl, "Open Simulation Interface: A generic interface for the environment perception of automated driving functions in virtual scenarios." 2017, Accessed: 2020/09/01.

[17] ISO 23150:2021, "Road vehicles - Data communication between sensors and data fusion unit for automated driving functions Logical interface," International Organization for Standardization (ISO), Geneva, Switzerland, 2021.

[18] D. Nuss, S. Reuter, M. Konrad, M. Munz, and K. Dietmayer, "Using grid maps to reduce the number of false positive measurements in advanced driver assistance systems," in 2012 15th International IEEE Conference on Intelligent Transportation Systems, 2012, pp. 15091514, ISSN: 2153-0017. 
[19] R. Priemer, Introductory Signal Processing, ser. Advanced Series In Electrical And Computer Engineering. World Scientific Publishing Company, 1990.

[20] S. Riedmaier, J. Schneider, B. Danquah, and F. Diermeyer, "Nondeterministic model validation methodology for simulation-based safety assessment of automated vehicles," Simulation Modelling Practice and Theory, 2021.

[21] T. Hanke, N. Hirsenkorn, B. Dehlink, A. Rauch, R. Rasshofer, and E. Biebl, "Classification of sensor errors for the statistical simulation of environmental perception in automated driving systems," in 2016 IEEE 19th International Conference on Intelligent Transportation Systems (ITSC). IEEE, 2016, pp. 643-648.

[22] H. Haferkorn, Optik: Physikalisch-technische Grundlagen und Anwendungen, 4th ed. Weinheim: Wiley-VCH, 2003.

[23] W. Menzel, "Antennas in automobile radar," in Handbook of Antenna Technologies, Z. N. Chen, Ed. Springer Singapore, 2014, pp. 1-22.

[24] H. Gotzig and G. Geduld, "Automotive LIDAR," in Handbook of Driver Assistance Systems: Basic Information, Components and Systems for Active Safety and Comfort, H. Winner, S. Hakuli, F. Lotz, and C. Singer, Eds. Springer International Publishing, 2016, pp. 405-430.

[25] H. Winner, "Automotive RADAR," in Handbook of Driver Assistance Systems: Basic Information, Components and Systems for Active Safety and Comfort, H. Winner, S. Hakuli, F. Lotz, and C. Singer, Eds. Springer International Publishing, 2016, pp. 325-403.

[26] K. Y. Yang, J. Skarda, M. Cotrufo, A. Dutt, G. H. Ahn, M. Sawaby, D. Vercruysse, A. Arbabian, S. Fan, A. Alù, and J. Vučković, "Inverse-designed non-reciprocal pulse router for chip-based lidar," Nature Photonics, vol. 14, no. 6, pp. 369-374, 2020.

[27] O. Toker and M. Brinkmann, "A novel nonlinearity correction algorithm for FMCW radar systems for optimal range accuracy and improved multitarget detection capability," Electronics, vol. 8, no. 11, p. $1290,2019$.

[28] L. Huang, H. Chen, and J. Bai, "Simulation of the effect of signal source's phase noise on millimeter wave automotive radar system based on SystemVue," in 2016 IEEE International Workshop on Electromagnetics: Applications and Student Innovation Competition (iWEM). IEEE, 2016, pp. 1-3.

[29] M. Dudek, I. Nasr, D. Kissinger, R. Weigel, and G. Fischer, "The impact of phase noise parameters on target signal detection in FMCW-radar system simulations for automotive applications," in Proceedings of 2011 IEEE CIE International Conference on Radar. IEEE, 2011, pp. 494-497.

[30] M. Hebel, M. Hammer, M. Arens, and A. L. Diehm, "Mitigation of crosstalk effects in multi-LiDAR configurations," in Electro-Optical Remote Sensing XII. Proc. SPIE 10796, 2018.

[31] S. Alland, W. Stark, M. Ali, and M. Hegde, "Interference in automotive radar systems: Characteristics, mitigation techniques, and current and future research," IEEE Signal Processing Magazine, vol. 36, no. 5, pp. 45-59, 2019.

[32] D. Hertel, "Extended use of incremental signal-to-noise ratio as reliability criterion for multiple-slope wide-dynamic-range image capture," Journal of Electronic Imaging, vol. 19, no. 1, 2010.

[33] L. Zhang, J. Chang, H. Li, Z. X. Liu, S. Zhang, and R. Mao, "Noise reduction of LiDAR signal via local mean decomposition combined with improved thresholding method," IEEE Access, vol. 8, pp. $113943-113952,2020$.

[34] A. Kukko, S. Kaasalainen, and P. Litkey, "Effect of incidence angle on laser scanner intensity and surface data," Applied Optics, vol. 47, no. 7, p. 986, 2008.

[35] A. Kamann, P. Held, F. Perras, P. Zaumseil, T. Brandmeier, and U. T. Schwarz, "Automotive radar multipath propagation in uncertain environments," in 21st International Conference on Intelligent Transportation Systems (ITSC), 2018, p. 6.

[36] A. A. Maradudin and E. R. Méndez, "Light scattering from randomly rough surfaces," Science Progress, vol. 90, no. 4, pp. 161-221, 2007.

[37] M. Colomb, P. Duthon, and F. Bernardin, "Spectral reflectance characterization of the road environment to optimize the choice of autonomous vehicle sensors," in 2019 IEEE Intelligent Transportation Systems Conference (ITSC). IEEE, 2019, pp. 1085-1090.

[38] R. Schneider, "Modellierung der Wellenausbreitung für ein bildgebendes Kfz-Radar," PhD Thesis, Universität Fridericana Karlsruhe, 1998.

[39] W. Y. Yan, A. Shaker, A. Habib, and A. P. Kersting, "Improving classification accuracy of airborne LiDAR intensity data by geometric calibration and radiometric correction," ISPRS Journal of Photogrammetry and Remote Sensing, vol. 67, pp. 35-44, 2012.

[40] T. Oguchi, "Electromagnetic wave propagation and scattering in rain and other hydrometeors," Proceedings of the IEEE, vol. 71, no. 9, pp. 1029-1078, 1983.

[41] H. Zhang, W. Hong, J. Xu, and Y. Zhu, "Research on automotive windshield impact on the w-band millimeter-wave transmission," in Asia-Pacific Microwave Conference (APMC). IEEE, 2015.

[42] L. Elterman and A. F. C. R. L. (U.S.), UV, Visible, and IR Attenuation for Altitudes to $50 \mathrm{Km}, 1968$, ser. Environmental research papers. Air Force Cambridge Research Laboratories, Office of Aerospace Research, United States Air Force, 1968

[43] S. Hasirlioglu, I. Doric, C. Lauerer, and T. Brandmeier, "Modeling and simulation of rain for the test of automotive sensor systems," in 2016 IEEE Intelligent Vehicles Symposium (IV). IEEE, 2016, pp. 286-291.

[44] T. Yamawaki and S. Yamano, "60-GHz millimeter-wave automotive radar," Fujitsu Ten Tech. J. 11, pp. 3-14, 1998.

[45] P. C. Barnum, S. Narasimhan, and T. Kanade, "Analysis of rain and snow in frequency space," International Journal of Computer Vision, vol. 86, no. 2-3, pp. 256-274, 2010.

[46] R. Nebuloni and C. Capsoni, "Laser attenuation by falling snow," in 2008 6th International Symposium on Communication Systems, Networks and Digital Signal Processing. IEEE, 2008, pp. 265-269.

[47] J. R. Kendra, K. Sarabandi, and F. T. Ulaby, "Radar measurements of snow: experiment and analysis," IEEE Transactions on Geoscience and Remote Sensing, vol. 36, no. 3, pp. 864-879, 1998.

[48] S. Hasirlioglu, I. Doric, A. Kamann, and A. Riener, "Reproducible fog simulation for testing automotive surround sensors," in 85th Vehicular Technology Conference (VTC). IEEE, 2017.

[49] H. Chandel and S. Vatta, "Occlusion detection and handling: A review," International Journal of Computer Applications, vol. 120, no. 10, pp. 33-38, 2015.

[50] J. Sun, Y. Cao, Q. A. Chen, and Z. M. Mao, "Towards robust LiDAR-based perception in autonomous driving: General black-box adversarial sensor attack and countermeasures," in Proceedings of the 29th USENIX Security Symposium, 2020, pp. 877-894.

[51] F. J. Duarte, "Lasers and their emission characteristics," in Tunable Laser Optics. Elsevier, 2003, pp. 177-201.

[52] T. Visentin, J. Hasch, and T. Zwick, "Analysis of multipath and DOA detection using a fully polarimetric automotive radar," in Proceedings of the 14th European Radar Conference, 2017, p. 4.

[53] M. I. Skolnik, Introduction to radar systems, 3rd ed., ser. McGrawHill electrical engineering series. McGraw-Hill, 2001.

[54] T. Li, C. Wang, L. Shi, M. Gao, and X. Fu, "The performance analysis of micro-doppler extracted from radar echoes under different bandwidth," in IET International Radar Conference 2013. Institution of Engineering and Technology.

[55] C. Chen, Q. Chen, J. Xu, and V. Koltun, "Learning to see in the dark," in Conference on Computer Vision and Pattern Recognition (CVPR 2018), 2018.

[56] S. Muckenhuber, H. Holzer, and Z. Bockaj, "Automotive Lidar Modelling Approach Based on Material Properties and Lidar Capabilities," Sensors, vol. 20, no. 11, 2020.

[57] C. Ricolfe-Viala and A.-J. Sánchez-Salmerón, "Correcting non-linear lens distortion in cameras without using a model," Optics \& Laser Technology, vol. 42, no. 4, pp. 628-639, 2010.

[58] M. P. Bristow, "Lidar-signal compression by photomultiplier gain modulation: influence of detector nonlinearity," Applied Optics, vol. 37, no. 27, pp. 6468-6479, Sep 1998.

[59] F. Hau, F. Baumgärtner, and M. Vossiek, "The degradation of automotive radar sensor signals caused by vehicle vibrations and other nonlinear movements," Sensors, vol. 20, no. 21, p. 6195, 2020.

[60] R. Lukac, "Single-sensor digital color imaging fundamentals," in Single-sensor imaging: Methods and applications for digital cameras, ser. Image processing series, R. Lukac, Ed. Boca Raton, FL: CRC Press, 2008.

[61] Q. Hao, J. Cao, Y. Hu, Y. Yang, K. Li, and T. Li, "Differential opticalpath approach to improve signal-to-noise ratio of pulsed-laser range finding," Optics Express, vol. 22, no. 1, pp. 563-575, 2014.

[62] A. W. Doerry, "Noise and noise figure for radar receivers," Sandia National Laboratories Report, no. SAND2016-9649, 2016.

[63] K. Hirakawa, "Color filter array image analysis for joint demosaicking and denoising," in Single-sensor imaging: Methods and applica- 
tions for digital cameras, ser. Image processing series, R. Lukac, Ed. Boca Raton, FL: CRC Press, 2008

[64] Z. Liu, W. Hunt, M. Vaughan, C. Hostetler, M. McGill, K. Powell, D. Winker, and Y. Hu, "Estimating random errors due to shot noise in backscatter lidar observations," Applied Optics, vol. 45, no. 48, pp. 4437-4447, 2006.

[65] H.-Y. Lin, K.-J. Li, and C.-H. Chang, "Vehicle speed detection from a single motion blurred image," Image and Vision Computing, vol. 26, pp. 1327-1337, 2008

[66] L. Gröll and A. Kapp, "Effect of fast motion on range images acquired by lidar scanners for automotive applications," IEEE Transactions on Signal Processing, vol. 55, no. 6, pp. 2945-2953, 2007.

[67] D. H. Marimont and B. A. Wandell, "Matching color images: the effects of axial chromatic aberration," Journal of the Optical Society of America A, vol. 11, no. 12, p. 3113, 1994.

[68] A. V. Pravdivtsev, "Simulation and assessment of stray light effects in infrared cameras using non-sequential ray tracing," Infrared Physics, vol. 60, pp. 306-311, 2013

[69] D. D. Lichti, S. J. Gordon, T. Tipdecho, and S. Lecturer, "Error models and propagation in directly georeferenced terrestrial laser scanner networks," Journal of Surveying Engineering, vol. 131, no. 1, pp. 135-142, 2005.

[70] L. Ray and M. Kulkarni, "Computation and correction of dynamic white balance in the automotive cameras," in 2019 IEEE International WIE Conference on Electrical and Computer Engineering (WIECON-ECE). IEEE, 2019, pp. 1-6.

[71] ISO 12232:2019, "Photography - Digital still cameras - Determination of exposure index, ISO speed ratings, standard output sensitivity, and recommended exposure index," International Organization for Standardization (ISO), Geneva, Switzerland, 2019.

[72] R. M. Measures, Laser remote sensing: Fundamentals and applications. New York, NY: Wiley, 1984.

[73] I. Jatzkowski, D. Wilke, and M. Maurer, "A deep-learning approach for the detection of overexposure in automotive camera images,' in 2018 21st International Conference on Intelligent Transportation Systems (ITSC). IEEE, 2018, pp. 2030-2035.

[74] G. P. Kokhanenko, I. E. Penner, and V. S. Shamanaev, "Expanding the dynamic range of a lidar receiver by the method of dynode-signal collection," Applied Optics, vol. 41, no. 24, p. 5073, 2002.

[75] R. Palum, "Optical antialiasing filters," in Single-sensor imaging. Methods and applications for digital cameras, ser. Image processing series, R. Lukac, Ed. Boca Raton, FL: CRC Press, 2008.

[76] I. Maksymova, C. Steger, and N. Druml, "Review of LiDAR Sensor Data Acquisition and Compression for Automotive Applications," Proceedings, vol. 2, no. 13, p. 852, Dec. 2018.

[77] K. Li, B. Habtemariam, R. Tharmarasa, and T. Kirubarjan, "Multitarget tracking with doppler ambiguity," IEEE Transactions on Aerospace and Eelectronic Systems, vol. 49, no. 4, p. 17, 2013.

[78] B. Höfle and N. Pfeifer, "Correction of laser scanning intensity data: Data and model-driven approaches," ISPRS Journal of Photogram metry and Remote Sensing, vol. 62, no. 6, pp. 415-433, 2007.

[79] M. Punke, S. Menzel, B. Werthessen, N. Stache, and M. Höpfl, "Automotive Camera (Hardware)," in Handbook of Driver Assistance Systems: Basic Information, Components and Systems for Active Safety and Comfort, H. Winner, S. Hakuli, F. Lotz, and C. Singer, Eds. Cham: Springer International Publishing, 2016, pp. 431-460.

[80] P. Bhargava, T. Kim, C. V. Poulton, J. Notaros, A. Yaacobi, E. Timurdogan, C. Baiocco, N. Fahrenkopf, S. Kruger, T. Ngai, Y. Timalsina, M. R. Watts, and V. Stojanović, "Fully integrated coherent lidar in 3d-integrated silicon photonics/65nm cmos," in 2019 Symposium on VLSI Circuits, 2019, pp. C262-C263.

[81] M. F. Holder, C. Linnhoff, P. Rosenberger, C. Popp, and H. Winner, "Modeling and simulation of radar sensor artifacts for virtual testing of autonomous driving," in 9. Tagung Automatisiertes Fahren, 2019.

[82] F. Pitié, A. Kokaram, and R. Dahyot, "Enhancement of digital photographs using color transfer techniques," in Single-sensor imaging: Methods and applications for digital cameras, ser. Image processing series, R. Lukac, Ed. Boca Raton, FL: CRC Press, 2008.

[83] B. Magnier, H. Abdulrahman, and P. Montesinos, "A review of supervised edge detection evaluation methods and an objective comparison of filtering gradient computations using hysteresis thresholds," Journal of Imaging, vol. 4, no. 6, p. 74, 2018.

[84] M. Buhren and B. Yang, "Simulation of automotive radar target lists considering clutter and limited resolution," in Proceedings of International Radar Symposium (IRS), 2007, pp. 195-200.
[85] H. Rohling and R. Mende, "OS CFAR performance in a $77 \mathrm{GHz}$ radar sensor for car application," in Proceedings of International Radar Conference, 1996, pp. 109-114.

[86] A. Mittal, S. Sofat, E. Hancock, and S. Mousset, "A statistical operator for detecting weak edges in low contrast images," in Image Analysis and Recognition, A. Campilho and M. Kamel, Eds. Berlin, Heidelberg: Springer Berlin Heidelberg, 2012, pp. 89-96.

[87] T. Ogawa, H. Sakai, Y. Suzuki, K. Takagi, and K. Morikawa, "Pedestrian detection and tracking using in-vehicle lidar for automotive application," in 2011 IEEE Intelligent Vehicles Symposium (IV). Baden-Baden, Germany: IEEE, Jun. 2011, pp. 734-739.

[88] J. Lombacher, M. Hahn, J. Dickmann, and C. Wohler, "Potential of radar for static object classification using deep learning methods," in IEEE MTT-S International Conference on Microwaves for Intelligent Mobility (ICMIM). San Diego, CA, USA: IEEE, May 2016.

[89] J. Moras, V. Cherfaoui, and P. Bonnifait, "A lidar perception scheme for intelligent vehicle navigation," in 11th International Conference on Control Automation Robotics \& Vision. IEEE, 2010, pp. 1809 1814.

[90] S. Heuel and H. Rohling, "Pedestrian classification in automotive radar systems," in 2012 13th International Radar Symposium. Warsaw, Poland: IEEE, May 2012, pp. 39-44.

[91] D. Kellner, J. Klappstein, and K. Dietmayer, "Grid-based DBSCAN for clustering extended objects in radar data," in 2012 IEEE Intelligent Vehicles Symposium. Alcal de Henares, Madrid, Spain: IEEE, Jun. 2012, pp. 365-370.

[92] M. Kronauge, C. Schroeder, and H. Rohling, "Radar target detection and doppler ambiguity resolution," in 11th Interantional Radar Symposium (IRS), 2010

[93] F. A. Alencar, L. A. Rosero, C. M. Filho, F. S. Osorio, and D. F. Wolf, "Fast Metric Tracking by Detection System: Radar Blob and Camera Fusion," in 2015 12th Latin American Robotics Symposium and 2015 3rd Brazilian Symposium on Robotics (LARS-SBR). Uberlandia, Brazil: IEEE, Oct. 2015, pp. 120-125.

[94] A. Palffy, J. Dong, J. F. P. Kooij, and D. M. Gavrila, "CNN Based Road User Detection Using the 3D Radar Cube," IEEE Robotics and Automation Letters, vol. 5, no. 2, pp. 1263-1270, Apr. 2020.

[95] M. Weber, M. Furst, and J. M. Zollner, "Direct 3d detection of vehicles in monocular images with a CNN based 3d decoder," in Intelligent Vehicles Symposium (IV). IEEE, 2019, pp. 417-423.

[96] S. Segal, A. Logvinenko, and A. Slapak, "Occlusion Handling in Radar for Detection of Obstacles Based on Tracking Model," in 2018 19th International Radar Symposium (IRS). Bonn, Germany: IEEE, Jun. 2018, pp. 1-10.

[97] C. Jacobs. (2020) From ADAS to driver replacement - is actual RADAR performance good enough.

[98] I.-S. Weon, S.-G. Lee, and J.-K. Ryu, "Object recognition based interpolation with $3 \mathrm{~d}$ LIDAR and vision for autonomous driving of an intelligent vehicle," IEEE Access, vol. 8, pp. 65 599-65 608, 2020.

[99] H. Uçar, "Radar cross section reduction," Journal of Naval Science and Engineering, vol. 9, no. 2, pp. 72-87, 2013.

100] H. Zhang, Z. Jiao, Y. Dong, and X. Li, "Evaluation of BRDF Archetypes for Representing Surface Reflectance Anisotropy Using MODIS BRDF Data," Remote Sensing, vol. 7, no. 6, pp. 7826-7845, 2015.

[101] Zhijian Li, L. Ligthart, Peikang Huang, Weining Lu, and W. van der Zwan, "Trade-off between sensitivity and dynamic range in designing digital radar receivers," in 2008 International Conference on $\mathrm{Mi}$ crowave and Millimeter Wave Technology. Nanjing, China: IEEE, Apr. 2008, pp. 1368-1371

[102] P. 1883:2020, "Operational Design Domain (ODD) taxonomy for an automated driving system (ADS) - Specification,” 2020.

[103] AIAG and VDA, FMEA Handbook - Failure Mode and Effects Analysis. Automotive Industry Action Group, 2019.

[104] Spirent, "Why Simulate?" 2010, Accessed: 2021/01/04. [Online]. Available: https://www.nottingham.ac.uk/grace/documents/resources/ glossariestutorials/aboutgnsssimulator.pdf

[105] Spirent, "Understanding Multipath and Obscuration," 2019, Accessed: 2021/01/04. [Online]. Available: https: //assets.ctfassets.net/wcxs9ap8i19s/6LwgGcbLrmTOJznVSzDYQ5/ 5bb618e5a809d1e848b5ceb4f9184c9b/ EB-Understanding-Multipath-and-Obscuration.pdf 